\title{
Lower hamstring extensibility in men compared to women is explained by differences in stretch tolerance
}

\author{
Paul WM Marshall ${ }^{*}$ and Jason C Siegler
}

\begin{abstract}
Background: This study examined whether passive hamstring tissue stiffness and/or stretch tolerance explain the relationship between sex and hamstring extensibility.

Methods: Ninety healthy participants, 45 men and 45 women (mean \pm SD; age $24.6 \pm 5.9$ years, height $1.72 \pm$ $0.09 \mathrm{~m}$, weight $74.6 \pm 14.1 \mathrm{~kg}$ ) volunteered for this study. The instrumented straight leg raise was used to determine hamstring extensibility and allow measurement of stiffness and stretch tolerance (visual analog pain score, VAS).

Results: Hamstring extensibility was $9.9^{\circ}$ greater in women compared to men $(p=0.003)$. VAS scores were $16 \mathrm{~mm}$ lower in women $(p=0.001)$. Maximal stiffness (maximal applied torque) was not different between men and women $(p=0.42)$. Passive stiffness (slope from $20-50^{\circ}$ hip flexion) was $0.09 \mathrm{Nm}^{0-1}$ lower in women $(p=0.025)$. For women, linear and stepwise regression showed that no predictor variables were associated with hamstring extensibility (adjusted $r^{2}=-0.03, p=0.61$ ). For men, $44 \%$ of the variance in hamstring extensibility was explained by VAS and maximal applied torque (adjusted $r^{2}=0.44, p<0.001$ ), with $41 \%$ of the model accounted for by the relationship between higher VAS scores and lower extensibility (standardized $\beta$ coefficient $=-0.64, p<0.001$ ).
\end{abstract}

Conclusions: The results of this study suggest that stretch tolerance and not passive stiffness explains hamstring extensibility, but this relationship is only manifest in men.

Keywords: Hamstring extensibility, Passive stiffness, Stretch tolerance

\section{Background}

Extensibility is defined as the ability of muscle tissue to lengthen or stretch beyond resting length. Lower hamstring extensibility is a functional characteristic of significant interest for the prevention and rehabilitation of locomotion related strain injuries [1,2], as well as the treatment of patients with chronic low back pain [3-5]. Passive stretching is a typical component of injury prevention and rehabilitation programs to address hamstring extensibility [6]. However, there is dispute as to the efficacy of passive stretching for improving hamstring extensibility. The equivocal evidence may, in part, be attributed to the use of different volumes of stretching prescribed between studies [7-9], small sample sizes, confusion about the mechanism of action for explaining changes in hamstring

\footnotetext{
* Correspondence: p.marshall@uws.edu.au

School of Science and Health, University of Western Sydney, Locked Bag 1797, Penrith South, NSW 2751, Australia
}

extensibility, and the confounding effect of small mixed sex groups [10-12]. Two factors typically measured for explaining changes in hamstring extensibility are passive stiffness and stretch tolerance. There is debate regarding the relative contribution of passive stiffness and stretch tolerance to hamstring extensibility. Moreover there is limited information examining similarities or differences between-sex in passive stiffness and stretch tolerance. The limited evidence examining between-sex differences contributes to the confusing evidence from training studies that use small mixed sex groups to examine changes in hamstring extensibility.

Muscle stiffness is the ratio of the change in torque to the change in muscle length, which may be examined during active or passive contractions [13]. Often measured during an instrumented passive straight leg raise (iSLR) test to allow observation of torque applied during stretch $[11,14,15]$, some research that has concluded passive 
stiffness does not explain extensibility, either acutely or following training $[8,10,11,16]$. However, these studies typically define passive stiffness from the maximal torque measured at the end of the hamstrings range of motion $[8,10,11,16]$. Maximal applied torque is probably not a valid estimation of passive muscle stiffness as the measurement does not take into account the ratio of the change in torque to change in muscle length during stretch. In contrast increased passive hamstring stiffness, quantified as the slope of the torque-angular position curve during passive hip flexion, was observed to significantly predict decreased extensibility in both healthy individuals and patients with chronic LBP [5]. Moreover, reductions in the slope of the torque-angular position curve were associated with improved hamstring extensibility following 4-weeks of passive stretching in healthy young men and women [12]. The reliance on measurement of maximal torque rather than examining changes in torque during the range of motion has contributed to the conclusion that hamstring extensibility is probably best explained by stretch tolerance.

Stretch tolerance is described as the willingness to tolerate the discomfort associated with stretch [11,17]. Typically, this has been self-reported as the angle during the range of motion where discomfort is reported $[8,17]$. One study suggested stretch tolerance does not explain extensibility, but no direct measure of stretch tolerance was performed during testing [5]. Other evidence that suggests stretch tolerance may contribute to hamstring extensibility has examined maximal perceived pain during an extensibility test before and after training interventions $[11,12]$. While increased hamstring extensibility was reported following passive stretch training programs $[11,12]$, maximal pain reported at the new end range of extensibility was not changed. This was interpreted in one study to mean that participants willingness to tolerate pain during stretch had improved [11], and was not discussed in the other [12]. Stretch tolerance does likely predict extensibility, but the contribution to extensibility as compared to changes in passive stiffness is unknown. Moreover, it is unclear whether there are between-sex differences in the contribution of stretch tolerance and passive stiffness to total hamstring extensibility.

One study using the iSLR reported no between-sex difference for extensibility, but lower passive stiffness for women [5]. This finding [5] is confounded by a small, mixed sample of healthy controls and LBP patients, and absence of a direct stretch tolerance measure. Other evidence using a prone knee flexion/extension perturbation model reported lower active and passive hamstring stiffness in women $[18,19]$. Thus it is reasonable to believe that passive stiffness will be lower in women, although it is not clear whether this will explain differences in extensibility during the iSLR. Moreover, it is unclear whether or not stretch tolerance will be lower in women concomitant to lower passive stiffness. Differences between men and women for pain sensitivity and tolerance have been reported [20]. Typically, these findings suggest that women have greater pain sensitivity and lower tolerance than men, which is often attributed to different hormonal profiles [20-22]. Whether women will self-report greater pain during stretch, when it is expected that passive stiffness will be lower and extensibility higher than men is unclear.

There is a need to understand whether the relationship between sex and hamstring extensibility is explained by hamstring passive tissue stiffness and stretch tolerance. Providing information about the factors that contribute to hamstring extensibility within each sex will help the design of targeted interventions. The purposes of this study were: 1) to compare measures of hamstring extensibility, passive stiffness, and stretch tolerance between men and women, 2) to examine the relationship between passive stiffness and stretch tolerance with hamstring extensibility within each sex.

\section{Methods \\ Participants}

Ninety participants, 45 men and 45 women, from a university population volunteered for this study (mean $\pm \mathrm{SD}$, men, age $23.4 \pm 4.5$ years, height $1.79 \pm 0.05 \mathrm{~m}$, mass 84.2 $\pm 10.3 \mathrm{~kg}$; women, age $25.7 \pm 6.9$ years, height $1.65 \pm$ $0.06 \mathrm{~m}$, weight $64.9 \pm 10.2 \mathrm{~kg}$ ). Men were taller and heavier $(\mathrm{p}<0.05)$. Participants were required to be free from any known metabolic and neuromuscular disease, or have any lifetime history of hamstring strain injury. Lifetime hamstring strain injury was defined as any hamstring injury that required absence from work, sport, training, or pain that required medication or treatment by a health care professional. No participant could recall an acute episode or period of back or hip pain where they had to seek medical treatment or miss a day of work or exercise. Current physical activity participation was collected from all participants via self-reporting $(n=42$ participants reported no regular physical activity apart from normal daily activities). For physically active participants, most reported performing various modes of exercise which did not make it possible to classify their training into one category for purposes of statistical analysis (e.g. yoga, stretching, resistance exercise). The two delineating factors between sexes for physical activity levels were that more men reported performing regular resistance exercise $(n=10$ reported between two to six resistance based sessions per week) compared to women $(n=3)$, while more women reported regular $(n=11)$ participation in group based exercise sessions (e.g. step aerobics, spin classes) compared to men $(n=0)$. Similar numbers of men $(n=12)$ and women $(\mathrm{n}=15)$ reported performing at least one weekly stretching session (e.g. home based stretching, stretching prior to 
sport training session, yoga or pilates class). Written informed consent was received from all participants. Ethical approval was received for this study from the University Human Research Ethics Committee.

\section{Instrumented straight leg raise (iSLR)}

The KinCom isokinetic dynamometer (Chattanooga, KinCom 125 Version 5.32) was used for iSLR testing. Participants were placed in a supine position on the examination table. They were fixed to the table by the use of straps across the trunk, pelvis, and opposite thigh. Adjustable support was provided to prevent the lumbar spine flattening onto the test surface during testing. The leg was fully extended in the apparatus and attached to the lever arm on the distal aspect of the thigh and mid-shank, and the ankle was fixed at $90^{\circ}$ of dorsiflexion. The center of rotation of the dynamometer lever arm was aligned with the hip joint center of rotation through the greater trochanter of the test limb. Following familiarization with the experimental set-up and preliminary movement through a partial range of motion (passive flexion of the hip performed by the investigator), the stop angle to be used during testing was established by passively flexing the participant's hip until they could no longer tolerate the stretch. This was performed between 3 to 5 times with $30 \mathrm{~s}$ rest between movements until a consistent maximum hip flexion angle (within $1^{\circ}$ hip flexion as observed from digital realtime output of the dynamometer software) was established. The maximum angle of these trials was set as the terminal limit of the dynamometer for the recorded trial. This procedure was used to ensure that a true maximum hip flexion angle was set, reducing the influence of participant choice on termination of range of motion. The hip was then passively flexed through the established range of motion by the dynamometer at a movement velocity of $5^{\circ}$. $\mathrm{sec}^{-1}$, which is similar to previous studies $[5,15,17]$.

\section{Hamstring extensibility}

Angular position of the lever arm and torque were continuously collected at $100 \mathrm{~Hz}$. Maximum hip flexion angle $\left(\right.$ leg $\left._{\text {max }}^{\circ}\right)$ in the sagittal plane was measured from output of the angular position of the lever arm relative to the initial starting position (leg fully extended in line with the trunk was calibrated to starting angle of $0^{\circ}$ ). Therefore $\mathrm{leg}_{\text {max }}^{\circ}$ was the dependent variable we measured and defined as representing hamstring extensibility. Inter-trial reliability within this study for $\operatorname{leg}_{\text {max }}^{\circ}$ during the iSLR was high (ICC $r=0.98)$. Intra-tester reliability for the iSLR has been reported (ICC $\mathrm{r}=0.94$, [23]), in addition to betweensession reliability $(\mathrm{ICC} \mathrm{r}=0.95,[12])$.

\section{Hamstring stiffness}

The active torque required to lift the leg was continuously recorded (Figure 1) and adjusted for limb weight, leg length, and the angular position of the lever arm. Stiffness was defined as the torque calculated at the different joint angles $(\mathrm{Me})$. The maximal value from the torque-time curve was recorded and defined as the maximal stiffness $\left(\mathrm{Me}_{\max }\right)$. The relative stiffness $\left(\mathrm{Me}_{\text {grad }}\right)$ through the common range of motion $\left(20\right.$ to $50^{\circ}$ based on hip flexion angle) was calculated from the slope of a linear trend line fitted to torque values recorded from 20 to $50^{\circ}$ hip flexion. For processing $\mathrm{Me}_{\text {grad, }}$ recorded torque values in $5^{\circ}$ increments $\left(20^{\circ}, 25^{\circ} \ldots . .45^{\circ}, 50^{\circ}\right)$ were calculated from the average 1-s torque recorded at each point in the range of motion (e.g. average torque recorded from 19.5 to $20.5^{\circ}$ was calculated for stiffness at $20^{\circ}$ hip flexion). Subsequently, the slope ( $\Delta$ torque/ $\Delta$ angular position; $\mathrm{Nm}^{\circ-1}$ ) and coefficient of determination $\left(r^{2}\right)$ to examine the goodness of fit were calculated from a linear line fit to the torque-angular position relationship for each participant. The common range of motion is achievable in healthy and clinical populations [5,24]. The average coefficient of determination for the linear line for participants was $r^{2}=0.94 \pm 0.01$ (not different between men and women). Analysis through the common range of motion allows comparison of passive tissue stiffness across a standard absolute range of motion between participants, independent of total extensibility or maximal applied torque, and is a measure that allows direct comparison across population cohorts with lower absolute extensibility (e.g. low back pain patients, athletes pre-season with low extensibility).

\section{Stretch tolerance}

The visual analog pain scale (VAS) was used to represent participant stretch tolerance. The VAS is a $100 \mathrm{~mm}$ horizontal line with "no pain" and "worst pain" anchored to the respective left $(0 \mathrm{~mm})$ and right $(100 \mathrm{~mm})$ ends. Participants were asked to draw a vertical line through the horizontal line at the point which best represented their maximal pain intensity experienced during the iSLR. The VAS score $(\mathrm{mm})$ was calculated by measuring the distance from "no pain" to their mark. The VAS score was collected immediately $(<5 \mathrm{~s})$ following iSLR testing. Between-trial reliability for VAS scores reported during the manual SLR tests to establish maximum range of motion (performed prior to the recorded iSLR) had an ICC $r$-value $>0.95$. Test-retest reliability of the VAS to measure self-rated pain has been demonstrated to be high with ICC scores between 0.70 and 0.83 [25]. The VAS has been estimated to be more sensitive to change than a verbal rating scale, and similar to an 11-point numeric rating scale [26].

\section{Statistical analysis}

Data were analyzed using SPSS v20 (Armonk NY, USA: IBM Corp). All data were normally distributed, as assessed 


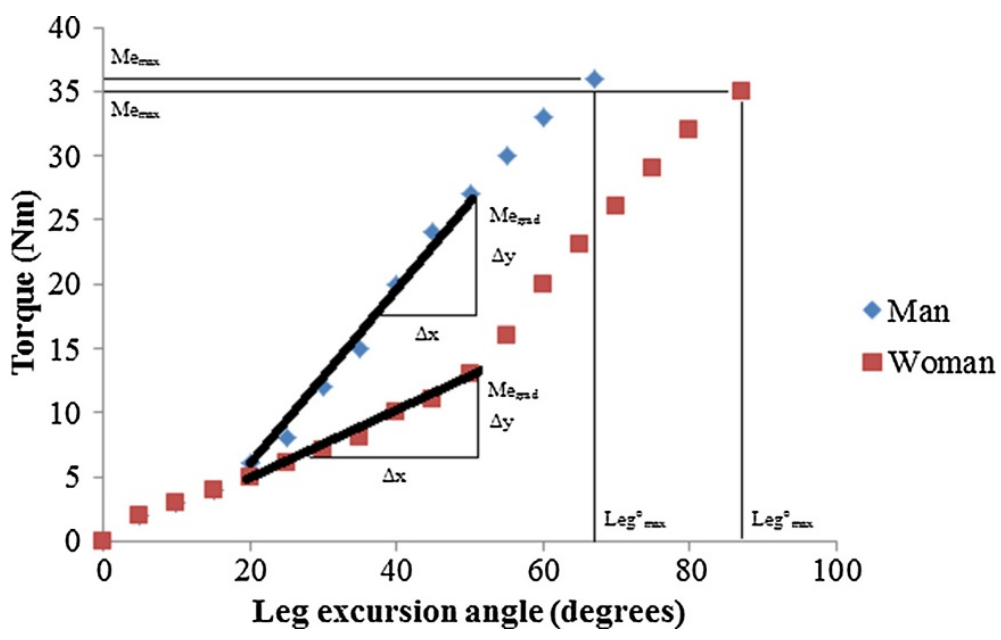

Figure 1 Representative results from a man and woman tested in this study for the torque measured during the instrumented straight leg raise test (iSLR). Passive tissue stiffness was measured as the slope $(\Delta y / \Delta x)$ through the common range of motion $\left(M e_{\text {grad, }}, 20-50^{\circ}\right)$, and peak torque applied during the iSLR ( $\mathrm{Me}_{\max }$ ). Maximum leg excursion angle (leg ${ }_{\max }$ ) was used to represent hamstring extensibility in this study (male,

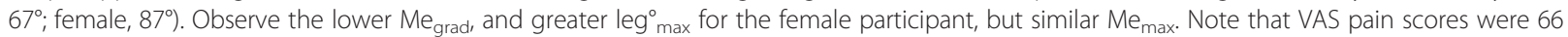
and $4 \mathrm{~mm}$ respectively for the male and female participant results presented here.

from inspection of the skewness and kurtosis of the data and Kolmogorov-Smirnov normality tests. Dependent variables were compared between men and women using independent samples t-tests. Mean differences and 95\% confidence intervals were calculated for between-sex differences. Pearson's correlation coefficients were calculated for within-sex relationships between the predictor variables $\mathrm{Me}_{\max }, \mathrm{Me}_{\text {grad, }}$ and VAS. Multiple linear and step-wise regression analyses were used to examine the relationship between $\mathrm{Me}_{\max }, \mathrm{Me}_{\text {grad, }}$ and VAS scores with $\mathrm{leg}_{\text {max. }}^{\circ}$ Regression analyses were performed for the entire sample, and separately for each sex. The significance level for this study was $\mathrm{p}<0.05$.

\section{Results}

Men and women results for iSLR variables are presented in Table $1 . \mathrm{Leg}_{\text {max }}^{\circ}$ was $9.9^{\circ}$ greater in women compared to men (95\% CI for mean difference, 3.5 to $16.3^{\circ}$, $\mathrm{p}=0.003)$. VAS scores were $16 \mathrm{~mm}$ lower in women ( $95 \% \mathrm{CI}$ for mean difference, -7 to $-26 \mathrm{~mm}, \mathrm{p}=0.001$ ). $\mathrm{Me}_{\text {grad }}$ was $0.09 \mathrm{Nm}^{.-1}$ lower in women $(95 \% \mathrm{CI}$ for mean difference, -0.01 to $\left.-0.16 \mathrm{Nm}^{.-1}, \mathrm{p}=0.025\right)$. No between-sex difference was observed for $\mathrm{Me}_{\max }$.

For women, correlation analysis identified no association between VAS and $\mathrm{Me}_{\max }(\mathrm{r}=0.19, \mathrm{p}=0.21)$ or $\mathrm{Me}_{\text {grad }}(\mathrm{r}=0.12, \mathrm{p}=0.41)$, and a significant positive association between the respective passive stiffness measures $(\mathrm{r}=0.47, \mathrm{p}=0.001)$. For men, there was a significant positive association between VAS and $\mathrm{Me}_{\max }(\mathrm{r}=0.66$, $\mathrm{p}<0.001$ ), but no association between VAS and Me grad $(r=0.11, p=0.47)$, or between the respective passive stiffness measures $(r=0.26, p=0.10)$.

For all participants, the predictor variables significantly explained $23 \%$ of the variance in $\mathrm{Leg}_{\text {max }}^{\circ}$ (adjusted $\mathrm{r}^{2}=$ $0.23, \mathrm{p}<0.001$ ). Stepwise regression identified VAS as the primary predictor of Leg $^{\circ}$ max (adjusted $\mathrm{r}^{2}=0.22$, $\mathrm{p}<0.001$ ), with higher VAS scores associated with lower $\mathrm{Leg}_{\text {max }}^{\circ}$ (standardized $\beta$ coefficient $=-0.48, \mathrm{p}<0.001$ ). For women, linear and stepwise regression showed that no predictor variables were associated with $\mathrm{Leg}^{\circ}{ }_{\text {max }}$ (Figure 2; adjusted $\mathrm{r}^{2}=-0.03, \mathrm{p}=0.61$ ). For men, $44 \%$ of the variance in $\mathrm{Leg}_{\text {max }}^{\circ}$ was explained by the predictor variables

Table 1 Between sex results (mean \pm SD, lower and upper limits for $95 \%$ confidence interval, CI) for iSLR measures of hamstring extensibility ( $\left(\mathrm{leg}_{\text {max }}^{\circ}\right)$, passive stiffness $\left(\mathrm{Me}_{\max }, \mathrm{Me}_{\text {grad }}\right)$, and stretch tolerance (hamstring VAS;mm)

\begin{tabular}{|c|c|c|c|c|c|}
\hline \multirow{2}{*}{ Variable } & \multicolumn{2}{|c|}{ Men $(n=45)$} & \multicolumn{2}{|c|}{ Women $(n=45)$} & \multirow{2}{*}{$\begin{array}{c}\mathrm{p}- \\
\text { value }\end{array}$} \\
\hline & Mean \pm SD & $95 \% \mathrm{Cl}$ & Mean \pm SD & $95 \% \mathrm{Cl}$ & \\
\hline $\operatorname{leg}_{\text {max }}^{\circ}\left({ }^{\circ}\right)$ & $79.4 \pm 17.8$ & 73.5 to 85.4 & $89.4 \pm 12.4$ & 85.2 to 93.5 & 0.003 \\
\hline $\mathrm{Me}_{\max }(\mathrm{Nm})$ & $43.6 \pm 23.6$ & 35.7 to 51.5 & $40.3 \pm 14.6$ & 35.4 to 45.1 & 0.42 \\
\hline $\mathrm{Me}_{\text {grad }}\left(\mathrm{Nm}^{\mathrm{o}^{-1}}\right)$ & $0.47 \pm 0.15$ & 0.42 to 0.53 & $0.38 \pm 0.21$ & 0.32 to 0.46 & 0.025 \\
\hline Hamstring VAS (mm) & $41 \pm 26$ & 32 to 49 & $24 \pm 19$ & 18 to 30 & 0.001 \\
\hline
\end{tabular}

Between-sex differences were observed for $\mathrm{leg}_{\text {max, }}^{\circ} \mathrm{Me}_{\text {grad, }}$ and VAS (t-test $\mathrm{p}$-values presented in table). 


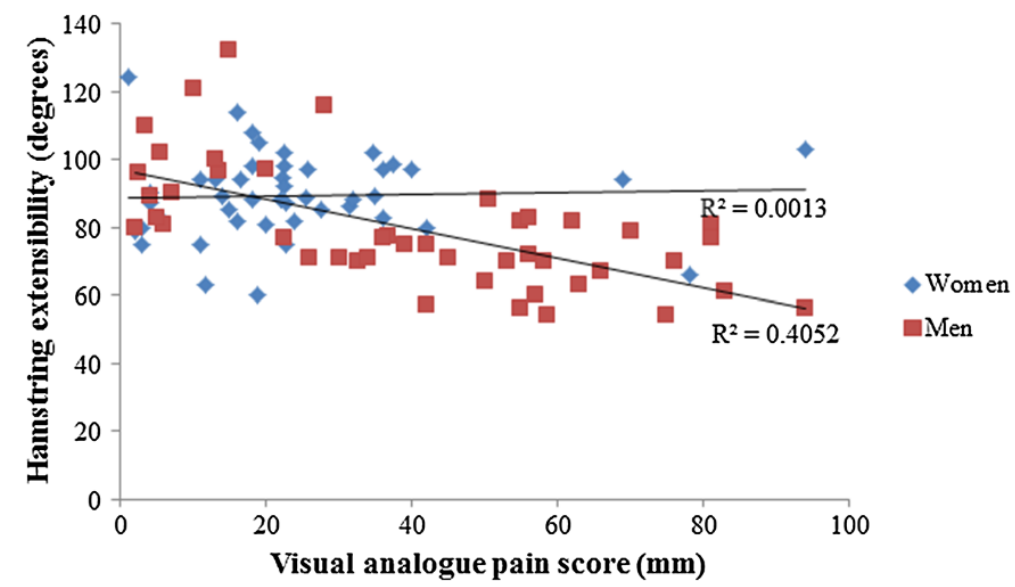

Figure 2 Relationship between hamstring extensibility (degrees; ${ }^{\circ}$ ) and visual analog pain scores (VAS; mm) for men $(n=45)$ and women (45) in this study. Trend lines are displayed for men and women, with a significant association between VAS and hamstring extensibility for men only (adjusted $r 2=0.41$, standardized $\beta$ coefficient $=-0.64, p<0.001$ ).

(adjusted $\mathrm{r}^{2}=0.44, \mathrm{p}<0.001$ ). Stepwise regression revealed that VAS explained $41 \%$ of variance, with higher VAS scores associated with lower Leg $_{\text {max }}^{\circ}$ (Figure 2; standardized $\beta$ coefficient $=-0.64, \mathrm{p}<0.001$ ), and $\mathrm{Me}_{\max }$ contributed a further $3 \%$ to the model with higher scores associated higher Leg $_{\text {max }}^{\circ}$ (standardized $\beta$ coefficient $=-0.32, \quad \mathrm{p}=$ 0.041).

\section{Discussion \\ Main findings}

The main findings of this study were 1) women had lower passive stiffness through a common range of motion, better stretch tolerance (lower VAS scores), and greater hamstring extensibility compared to men, and 2) stretch tolerance, but not passive stiffness, was a significant predictor of total hamstring extensibility for men only.

\section{Between sex differences in extensibility}

The results of this study support previous findings for between-sex differences in the mechanical properties of hamstring stiffness and extensibility $[18,19]$. Previous research has provided insight into mechanistic factors that may contribute to the between-sex differences reported here. One study reported that increased electromechanical delay (EMD) of the medial hamstrings during the iSLR test was associated with greater hamstring extensiblity in women, suggesting that the neuromuscular control of the hamstrings during stretch is altered in women [5]. Another study [18] reported that greater hamstring stiffness in men, as measured by an oscillating knee flexion/extension protocol, was positively associated with hamstring cross-sectional area (CSA). Measures of both stiffness and CSA were greater in male participants [18]. During the iSLR procedure in the current study, the applied torque to passively flex the hip was corrected for the limb weight, angular position, and lever arm of the participant. This calculation does not account for hamstring muscle CSA. Future research should examine whether passive stiffness, as measured by the iSLR method used in this study, may be explained by hamstring CSA and thus explain between-sex differences observed.

\section{The association between stretch tolerance and extensiblity}

The results of this study provide evidence that stretch tolerance, defined as the intensity of pain elicited during stretch, is the main explanatory variable of hamstring extensibilty as compared to measures of passive stiffness. This relationship was only observed for men, and not women. Lower VAS scores in women was unexpected, as previous findings suggest that women have higher pain sensitivity [20-22]. The self-report of pain, as performed in this study, is a complex interaction between physiological inputs, particularly nociceptive input, and behavioural interpretation. Higher pain scores in men may be associated with between sex differences in afferent feedback, particularly of the group III afferents. Group III afferents, also known as A $\delta$ fibers, are thinly myelinated fibers and have free nerve endings within the connective tissue of skeletal muscle [27]. Group III afferents primarily transmit information about mechanical stimuli in muscle [28], and in combination with group IV afferents are activated by nociceptive stimuli (e.g. bradykinin) and thus are the proposed source of pain in skeletal muscle. Research examining discharge properties of group III afferents in response to constant stretch is equivocal, but responses do increase as tension developed in a muscle increases [29]. It is not clear whether 
the pain scores reported in this study, particularly those reported by men, are mediated by increased group III afferent discharge rates. Moreover, there is no evidence examining group III afferent discharge properties during a stretching task where mechanical tension within the muscle is gradually increasing, or comparing group III discharge properties between sexes. Concomitant to nociception potentially explaining between sex differences in stretch tolerance is consideration for whether pain is causing a reflex response that impairs hamstring extensibility in men.

Johansson and Sojka proposed that muscle pain produces disturbances in proprioception, stiffness regulation, and motor control by altering stretch sensitivity and the discharge of spindle afferents via gamma fusimotor neurons [30]. Thus, it is reasonable to believe that increased pain during stretch may alter the neuromuscular control of the hamstrings, increase the eccentric contraction during stretch, and limit extensibility. Existing evidence would suggest that muscle activity does not contribute to termination of the straight leg raise. It was recently reported that surface EMG signals obtained from the hamstring muscles during the passive straight leg raise were not related to measures of stiffness or extensibility [5]. Indeed, healthy controls exhibited higher hamstring EMG signals as well as greater extensibility compared to patients with chronic low back pain, thus negating suggestions that increased muscle activity is detrimental during a passive straight leg raise. The physiological link between pain and increased spindle output has also been questioned. Recent evidence using experimentally induced muscle pain reported that muscle spindle output was not increased, suggesting that stimulation of group III and IV afferents fails to excite fusimotor neurons and increase muscle spindle discharge [31]. Therefore the causation for termination of the test and thus the relationship between pain and extensibility, evident only in men, is probably mediated by behavioural interpretation of the pain elicited during stretch.

\section{Application and limitations}

Recommendations for training interventions to improve hamstring extensibility in each sex cannot be made in this study. While current evidence would suggest that higher volumes of stretching (e.g. multiple hamstring stretches performed up to 5 times per week) are most effective for increasing extensibility and thus should be prescribed to both sexes $[7,12]$, it is not clear whether men also need additional intervention to improve their ability to tolerate stretch. The results of this study do suggest that findings and conclusions from previous literature exploring mechanisms of action for changing hamstring extensibility using small mixed sex samples are strongly confounded because of the differences between each sex for the relationship between stretch tolerance and extensibility.

There are several limitations to this study. First, while we recorded current physical activity levels, we did not record historical physical activity levels. Since the between-sex difference in extensibility was likely explained by pain and the behavioural response to pain, it is a noteworthy limitation that historical activity was not recorded. It is reasonable to believe that historical exposure to stretching, and types of activity where hamstring range of motion is trained (e.g. dance), could influence the familiarity with the type of stretch used in this study and thus the between-sex difference. Other muscles in the posterior kinetic chain (e.g. gluteals) likely contribute to the extensibility and stiffness measures in this study. Therefore, while the primary muscle stretch is applied to, these results are not specific to only the hamstrings. While not measured, we do not believe that hamstring extensibility was significantly affected by changes in the displacement or strain of the lumbosacral nerve roots during iSLR testing. By stabilizing the lumbar spine and using a fixed ankle position we have controlled these potentially confounding variables that have been observed to increase spinal nerve root tension [32]. Finally, the two measures of passive stiffness were based on the slope through the common range of motion (20 to $50^{\circ}$ hip flexion) and the maximal applied torque. Other researchers have applied a $4^{\text {th }}$ order polynomial to hamstring torque data during stretch, and have calculated the average first differential from a number of common points during the range of motion as the measure of stiffness (e.g. every $5^{\circ}$ during the final $15^{\circ}$ during the test [33]). Polynomial modelling may adequately fit the entire torque-angular position curve, but absolute application of a $4^{\text {th }}$ order polynomial for all participants assumes this is the best fit for the data. The passive stiffness measure in this study is based on the assumption of linearity between torque and angular position $\left(r^{2}=0.94 \pm 0.01\right)$ in a range of motion $\left(20\right.$ to $\left.50^{\circ}\right)$ that is achievable for healthy and chronic back pain populations. In future, methods should be applied that are individualized to each participant for curve-fit modelling and quantifying stiffness.

\section{Conclusions}

Greater hamstring extensibility in women was not associated with the lower passive stiffness and stretch tolerance scores. In contrast, lower extensibility in men was associated with higher pain scores. The results of this study suggest that stretch tolerance does explain hamstring extensibility, but this relationship is only manifest in men.

\section{Competing interests}

The authors declare that they have no competing interests. 


\section{Author contributions}

PM designed the study and coordinated data collection and analysis. PM and JS were both involved in the data analysis, preparation and editing of the final manuscript. Both authors read and approved the final manuscript.

\section{Acknowledgements}

Funding for this project and the publication was provided by internal funding sources in the School of Science and Health, University of Western Sydney.

Received: 10 March 2014 Accepted: 3 July 2014

Published: 7 July 2014

\section{References}

1. Witvrouw E, Danneels L, Asselman P, D'Have T, Cambier D: Muscle flexibility as a risk factor for developing muscle injuries in male professional soccer players. A prospective study. Am J Sports Med 2003, 31(1):41-46.

2. Bradley $P$, Portas $M$ : The relationship between preseason range of motion and muscle strain injury in elite soccer players. J Strength Cond Res 2007, 21:1155-1159.

3. Esola MA, McClure PW, Fitzgerald GK, Siegler S: Analysis of lumbar spine and hip motion during forward bending in subjects with and without a history of low back pain. Spine 1996, 21(1):71-78.

4. McHugh MP, Kremenic IJ, Fox MB, Gleim GW: The role of mechanical and neural restraints to joint range of motion during passive stretch. Med SCi Sports Exerc 1998, 30(6):928-932.

5. Marshall PWM, Mannion J, Murphy BA: Extensibility of the Hamstrings is best explained by mechanical components of muscle contraction, not behavioral measures in individuals with chronic low back pain. PM\&R 2009, 1:709-718.

6. Brooks JHM, Fuller CW, Kemp SPT, Reddin DB: Incidence, risk, and prevention of hamstring muscle injuries in professional rugby union. Am J Sports Med 2006, 34(8):1297-1306.

7. Ylinen J, Kautiainen $\mathrm{H}$, Hakkinen A: Comparison of active, manual, and instrumental straight leg raise in measuring hamstring extensibility. J Strength Cond Res 2010, 24(4):972-977.

8. Folpp H, Deall S, Harvey LA, Gwinn T: Can apparent increases in muscle extensibility with regular stretch be explained by changes in tolerance to stretch? Aus J Physiol 2006, 52(1):45-50

9. Roberts JM, Wilson K: Effect of stretching duration on active and passive range of motion in the lower extremity. Br J Sports Med 1999, 33:259-263.

10. Halbertsma JPK, Göeken LNH: Stretching exercises: effect on passive extensibility and stiffness in short hamstrings of healthy subjects. Arch Phys Med Rehabil 1994, 75(9):976-981

11. Law RYW, Harvey LA, Nicholas MK, Tonkin L, De Sousa M, Finniss DG: Stretch exercises increase tolerance to stretch in patients with chronic musculoskeletal pain: a randomized controlled trial. Phys Ther 2009, 89(10):1016-1026.

12. Marshall PWM, Cashman A, Cheema BS: A randomized controlled trial for the effect of passive stretching on measures of hamstring extensibility, passive stiffness, strength, and stretch tolerance. J Sci Med Sport 2011, 14(6):535-540.

13. McNair PJ, Wood GA, Marshall RN: Stiffness of the hamstring muscles and its relationship to function in anterior cruciate ligament deficient individuals. Clin Biomech 1992, 7:131-137.

14. Lee $\mathrm{R}$, Munn J: Passive moment about the hip in straight leg raising Clin Biomech 2000, 15(5):330-334

15. Tafazzoli F, Lamontagne M: Mechanical behaviour of hamstring muscles in low-back pain patients and control subjects. Clin Biomech 1996, 11(1):16-24

16. Magnusson SP, Simonsen EB, Aagaard P, Soørensen H, Kjær M: A mechanism for altered flexibility in human skeletal muscle. J Physiol 1996, 497(1):291-298.

17. Halbertsma JPK, Göeken LNH, Hof AL, Groothoff JW, Eisma WH: Extensibility and stiffness of the hamstrings in patients with nonspecific low back pain. Arch Phys Med Rehabil 2001, 82(2):232-238.

18. Blackburn T, Bell D, Norcross M, Hudson J, Kimsey M: Sex comparison of hamstring structural and material properties. Clin Biomech 2009, 24:65-70.
19. Blackburn T, Padua D, Riemann B, Guskiewicz K: The relationship between active extensibility, and passive and active stiffness of the knee flexors. J Electromyogr Kinesiol 2004, 14:683-691.

20. Wiesenfeld-Hallin Z: Sex differences in pain perception. Gend Med 2005 2(3):137-145.

21. Wise EA, Price DD, Myers CD, Heft MW, Robinson ME: Gender role expectations of pain: relationship to experimental pain perception. Pain 2002, 96(3):335-342.

22. Robinson ME, Riley JL, Myers CD, Papas RK, Waxenberg LB, Fillingim RB: Gender role expectations of pain: relationship to sex differences in pain. J Pain 2001, 2(5):251-257.

23. Ylinen J, Kankainen T, Kautiainen H, Rezasoltani A, Kuukkanen T, HÃakkinen A: Effect of stretching on hamstring muscle compliance. J Rehabil Med 2009, 41(1):80-84.

24. McHugh MP, Connolly DAJ, Eston RG, Kremenic IJ, Nicholas SJ, Gleim GW: The role of passive muscle stiffness in symptoms of exercise-induced muscle damage. Am J Sports Med 1999, 27(5):594-599.

25. Li L, Liu X, Herr K: Postoperative pain intensity assessment: a comparison of four scales in Chinese adults. Pain Med 2007, 8(3):223-234.

26. Breivik EK, Björnsson GA, Skovlund E: A comparison of pain rating scales by sampling from clinical trial data. Clin J Pain 2000, 16(1):22-28.

27. von During $M$, Andres $\mathrm{KH}$ : Topography and usltrastructure of group III and IV nerve terminals of cat's gastrocnemius-soleus muscle. In The primary afferent neuron: a survey of recent morpho-functional aspects. Edited by Zenker W, Neuhuber WL. New York: Plenum; 1990:35-41.

28. Hayes SG, Kaufman MP: Gadolinium attenuates exercise pressor reflex in cats. Am J Physiol Heart Circ Physiol 2001, 280:H2153-2161.

29. Kaufman M, Hayes SG, Adreani CM, Pickar JG: Discharge properties of group III and IV muscle afferents. In Sensorimotor contrl of movement and posture. Edited by Gandevia SC, Proske U, Stuart DG. New York: Springer US; 2002

30. Johansson H, Sojka P: Pathophysiological mechanisms involved in genesis and spread of muscular tension in occupational muscle pain and in chronic musculoskeletal pain syndromes: a hypothesis. Med Hypotheses 1991, 35(3):196-203.

31. Fazalbhoy A, Macefield VG, Birznieks I: Tonic muscle pain does not increase fusimotor drive to the human leg muscles: implications for chronic muscle pain. Exp Physiol 2013, 98(6):1125-1132.

32. Gilbert KK, Brismee JM, Collins DL, James CR, Shah RV, Sawyer SF, Sizer PS: 2006 Young investigator award winner: Lumbosacral nerve root displacement and strain: Part 2. A comparison of 2 straight leg raise conditions in unembalmed cadavers. Spine 2007, 32(14):1521-1525.

33. Palmer TB, Jenkins NDM, Cramer JT: Reliability of manual versus automated techniques for assessing passive stiffness of the posterior muscles of the hip and thigh. J Sports Sci 2013, 31(8):867-877.

\section{doi:10.1186/1471-2474-15-223}

Cite this article as: Marshall and Siegler: Lower hamstring extensibility in men compared to women is explained by differences in stretch tolerance. BMC Musculoskeletal Disorders 2014 15:223.

\section{Submit your next manuscript to BioMed Central and take full advantage of:}

- Convenient online submission

- Thorough peer review

- No space constraints or color figure charges

- Immediate publication on acceptance

- Inclusion in PubMed, CAS, Scopus and Google Scholar

- Research which is freely available for redistribution 